\title{
Self-energy effects in cuprates and the dome-shaped behavior of the superconducting critical temperature
}

\author{
Guillermo Buzon, Adriana Foussats, Matías Bejas, and Andrés Greco \\ Facultad de Ciencias Exactas, Ingeniería y Agrimensura and Instituto de \\ Física Rosario (UNR-CONICET). Av. Pellegrini 250-2000 Rosario-Argentina
}

(Dated: August 14, 2018)

\begin{abstract}
Hole doped cuprates show a superconducting critical temperature $T_{c}$ which follows an universal dome-shaped behavior as function of doping. It is believed that the origin of superconductivity in cuprates is entangled with the physics of the pseudogap phase. An open discussion is whether the source of superconductivity is the same that causes the pseudogap properties. The $t$ - $J$ model treated in large- $\mathrm{N}$ expansion shows $d$-wave superconductivity triggered by non-retarded interactions, and an instability of the paramagnetic state to a flux phase or $d$-wave charge density wave $(d$-CDW) state. In this paper we show that self-energy effects near $d$-CDW instability may lead to a dome-shaped behavior of $T_{c}$. In addition, it is also shown that these self-energy contributions may describe several properties observed in the pseudogap phase. In this picture, although fluctuations responsible for the pseudogap properties leads to a dome-shaped behavior, they are not involved in pairing which is mainly non-retarded.

PACS numbers: 74.72.-h, 74.72.Gh, 74.72.Kf, 74.25.Dw
\end{abstract}

\section{INTRODUCTION}

In spite of many years of research the origin of superconductivity in cuprates is still controversial. The superconducting critical temperature $\left(T_{c}\right)$ follows an universal dome-shaped behavior (DSB) where its maximum value takes place at optimal doping $(\delta \sim 0.16)$, which separates underdoped (UD) from overdoped (OD) regions. Another universal feature is the $d$-wave symmetry of the superconducting gap which has a maximum on the Fermi surface (FS) point in the $(0,0)-(0, \pi)$ direction (antinodal direction) and vanishes upon approaching the Brillouin zone diagonal (nodal direction) $\stackrel{1}{=}$ In addition, UD and OD regions show very distinct normal state properties as observed, for instance, by angle-resolved photoemission spectroscopy (ARPES) and transport experiments. Several macroscopic and microscopic experiments support the point of view that in UD a pseudogap (PG) appears in the normal state at a temperature $T^{*}$ which is well above $T_{c}$ and, in contrast to $T_{c}$, increases with decreasing doping. 1

In spite of the consensus about the existence of the $\mathrm{PG}$, its origin and nature remain elusive. Two major scenarios were proposed to explain the PG. In one scenario it originates from preformed pairs above $T_{c} \stackrel{\underline{2}}{\underline{-4}}$ In the second one, the PG is distinct from the superconducting gap and associated with a certain order which competes with superconductivity $\underline{\underline{5}-\underline{\underline{7}}}$ Several phenomenological models for the two-gap scenario have been proposed, but invoking different orders: $d$-wave charge density wave $(d$-CDW $), \stackrel{8}{,} d$-wave Fermi surface deformations, $\frac{9}{,}$ charge density wave $\underline{10}-13$ including stripes, $\stackrel{14,15}{\underline{1}}$ phase separation, $, 10,11,16$ and others such as resonating-valencebond-type charge order ${ }^{17}$ and loop-current order ${ }^{18,19}$

One dispute about the origin of superconductivity is whether pairing is originated by non-retarded or retarded interactions $\stackrel{20}{=}$ In the latter case, as in the electron- phonon (e-ph) conventional superconductivity, the excitations responsible for the pairing glue should be also present in transport and dynamical properties in the normal state (see Ref. 21] and references therein). On the other side, in the former case transport and dynamical properties in the normal state are not affected by the pairing interaction. ${ }^{22}$ Non-retarded interactions were proposed earlier ${ }^{23}$ and still firmly supported $\stackrel{24}{ }$ Recent experiments suggest that pairing may be certainly nonretarded $: 25,26$

The common belief is that superconductivity is entangled with the PG physics. Thus, a model candidate for describing cuprates must show $d$-wave superconductivity following a DSB and its relation with properties observed in the PG phase. The $t-J$ model treated in large$\mathrm{N}$ expansions shows, at low doping and low temperature, tendencies to several kinds of instabilities, being the flux phase (FP) the leading one ${ }^{27-29}$ Below a transition temperature $T_{F P}$ the $\mathrm{FP}$ is developed and the translational symmetry broken. The FP can be associated to the $d$-CDW phase which was proposed phenomenologically for describing the PG phase in cuprates ${ }^{\frac{8}{}} d$-CDW and FP are characterized by orbital currents flowing in a staggered pattern around each plaquette. Although the FP bears several characteristics for being considered as a candidate for the PG, the breaking of the translational symmetry and the existence of a phase transition are not clear from the experiments. On the other hand, non-retarded $d$-wave superconductivity triggered by the antiferromagnetic exchange $J$ is also observed in the $t$ $J$ model but with $T_{c}$ that monotonically increases with decreasing doping $2 \underline{28,30}$

The path integral large- $\mathrm{N}$ approach for the $t-J$ model in the context of Hubbard operators, $\frac{31}{2}$ allows the computation of fluctuations above the mean-field level and the calculation of self-energy effects $\underline{32}$ These self-energy effects were recently confronted with experiments in the 
normal state, such as angle-dependent magnetoresistance (ADMR) 33 and ARPES, $34-38$ after which they show qualitative agreement. We have shown that several aspects related to the Fermi arc phenomenology and ADMR features can be described considering self-energy effects near the FP instability.

In this paper we discuss the possibility that in the proximity to the FP instability, self-energy effects and nonretarded paring play together and develop a DSB for $T_{c}$. At the same time this process may describe several normal state properties observed in the pseudogap phase.

The paper is organized as follows. In Sec. [I] we review the background supporting present calculation. The FP instability of the paramagnetic phase, the existence of a quantum critical point (QCP), and the main characteristics of the dynamical flux susceptibility are presented. In Sec. III self-energy effects in the proximity to the flux instability, their role on normal state properties as those observed in ARPES and ADMR, and their importance for describing the pseudogap features are discussed. In Sec. IV the interplay between SC and the self-energy associated with the PG is discussed. Discussion and conclusion are presented in Sec. V.

\section{BACKGROUND}

The $t-J$ model, derived from the Hubbard model in the strong coupling limit,,$\underline{39}$ is considered one of the basic models for studying correlated systems as high- $T_{c}$ cuprates. The model is described by the Hamiltonian

$$
H=-\sum_{i, j, \sigma} t_{i j} \tilde{c}_{i \sigma}^{\dagger} \tilde{c}_{j \sigma}+J \sum_{<i, j>}\left(\vec{S}_{i} \cdot \vec{S}_{j}-\frac{1}{4} n_{i} n_{j}\right)
$$

where $t_{i j}=t\left(t^{\prime}\right)$ is the hopping integral between the first (second) nearest-neighbor sites on a square lattice, $J$ is the Heisenberg exchange interaction between nearestneighbor sites, $n_{i}$ is the electron density operator and $\vec{S}_{i}$ is the spin. $\tilde{c}_{i \sigma}^{\dagger}$ and $\tilde{c}_{i \sigma}$ are the creation and annihilation operators of electrons with spin $\sigma(\sigma=\downarrow, \uparrow)$ respectively, under the constraint that double occupancy of electrons is excluded at any site $i$.

Although the simple appearance of this model, $\tilde{c}_{i \sigma}^{\dagger}$ and $\tilde{c}_{i \sigma}$ are Hubbard operators which makes the treatment highly nontrivial. $\stackrel{40}{ }$ Several numerical methods have been applied for studying this model. Among others stand: the Quantum Monte-Carlo, which is suitable for calculating spectral functions for one-hole case, $\stackrel{41}{\sharp}$ while for finite doping the problem of the sign makes the calculation uncontrollable; and the Lanczos diagonalization ${ }^{42}$ and its finite temperature version $\underline{43}$ which are limited to finite clusters. Among the analytical methods one of the most popular is the slave-boson approximation where $\tilde{c}_{i \sigma}^{\dagger}$ and $\tilde{c}_{i \sigma}$ are decoupled in terms of fictitious particles $\underline{\underline{44}}$ However, the decoupling scheme introduces a gauge degree of freedom which requires gauge fixing and complicates the treatment of fluctuations above mean-field level ${ }^{44,45}$ See Ref. [46] for recent discussion about the interest in analytical computation of self-energy fluctuations.

In order to avoid these difficulties, a large- $\mathrm{N}$ expansion for the $t$ - $J$ model was formulated in terms of a path integral for Hubbard operators. In this formulation $\tilde{c}_{i \sigma}^{\dagger}$ and $\tilde{c}_{i \sigma}$ are treated without invoking fictitious particles. In the large- $\mathrm{N}$ approach the two spin components are extended to $\mathrm{N}$ and an expansion in powers of the small parameter $1 / \mathrm{N}$ is performed. In spite that the physical spin projection is two and not $\mathrm{N}$, the large- $\mathrm{N}$ expansion provides a controllable framework with no perturbative expansions in any model parameter, by which the results are in strong coupling. On the other hand, results obtained in large- $\mathrm{N}$ are improved with increasing doping: ${ }^{32}$ This large- $N$ treatment yields, in the square lattice, to a paramagnetic state with a quasiparticle (QP) dispersion

$$
\begin{aligned}
\epsilon_{\mathbf{k}}= & -(t \delta+r J)\left[\cos \left(k_{x}\right)+\cos \left(k_{y}\right)\right] \\
& +2 t^{\prime} \delta \cos \left(k_{x}\right) \cos \left(k_{y}\right)-\mu,
\end{aligned}
$$

where the contribution $r$ to the mean-field band and the chemical potential $\mu$ must be obtained self-consistently 31 from

$$
r=\frac{1}{N_{s}} \sum_{\mathbf{k}} \cos \left(k_{x}\right) n_{F}\left(\epsilon_{\mathbf{k}}\right),
$$

and

$$
1-\delta=\frac{2}{N_{s}} \sum_{\mathbf{k}} n_{F}\left(\epsilon_{\mathbf{k}}\right),
$$

where $n_{F}$ is the Fermi function, $\delta$ is the doping away from half-filling, and $N_{s}$ is the number of sites.

The path integral large- $\mathrm{N}$ expansion allows to go beyond mean-field and to compute self-energy fluctuations in $\mathcal{O}(1 / N)$. The expression for the self-energy is ${ }^{32}$

$$
\begin{array}{r}
\operatorname{Im} \Sigma(\mathbf{k}, \omega)=-\frac{1}{N_{s}} \sum_{\mathbf{q}, a, b} h_{a}\left(\mathbf{k}, \mathbf{q}, \omega-\epsilon_{\mathbf{k}-\mathbf{q}}\right) h_{b}\left(\mathbf{k}, \mathbf{q}, \omega-\epsilon_{\mathbf{k}-\mathbf{q}}\right) \\
\times \operatorname{Im}\left[D_{a b}\left(\mathbf{q}, \omega-\epsilon_{\mathbf{k}-\mathbf{q}}\right)\right]\left[n_{F}\left(-\epsilon_{\mathbf{k}-\mathbf{q}}\right)+n_{B}\left(\omega-\epsilon_{\mathbf{k}-\mathbf{q}}\right)\right]
\end{array}
$$

where $n_{B}$ is the Bose factor, and the vector $h_{a}(\mathbf{k}, \mathbf{q}, \nu)$ is 


$$
\begin{aligned}
h_{a}(\mathbf{k}, \mathbf{q}, \nu)= & \left\{\frac{2 \epsilon_{\mathbf{k}-\mathbf{q}}+\nu+2 \mu}{2}+J r\left[\cos \left(k_{x}-\frac{q_{x}}{2}\right) \cos \left(\frac{q_{x}}{2}\right)+\cos \left(k_{y}-\frac{q_{y}}{2}\right) \cos \left(\frac{q_{y}}{2}\right)\right] ; 1 ;\right. \\
& \left.-J r \cos \left(k_{x}-\frac{q_{x}}{2}\right) ;-J r \cos \left(k_{y}-\frac{q_{y}}{2}\right) ; J r \sin \left(k_{x}-\frac{q_{x}}{2}\right) ; J r \sin \left(k_{y}-\frac{q_{y}}{2}\right)\right\} .
\end{aligned}
$$

The information contained in the vector $h_{a}(\mathbf{k}, \mathbf{q}, \nu)$ is the following: the first component corresponds to the usual charge channel, the second component corresponds to the non-double occupancy constraint, and the last four correspond to the Heisenberg exchange channels, thus, these exists only for finite $J$.

In Eq.(5) $D_{a b}$ is a $6 \times 6$ matrix which contains contributions from the six different channels and their mixing. $D_{a b}$ describes all possible types of charge susceptibilities 29 and can be written as

$$
D_{a b}^{-1}\left(\mathbf{q}, i \nu_{n}\right)=\left[D_{a b}^{(0)}\left(\mathbf{q}, i \nu_{n}\right)\right]^{-1}-\Pi_{a b}\left(\mathbf{q}, i \nu_{n}\right)
$$

where

$$
D_{a b}^{(0)}\left(\mathbf{q}, i \nu_{n}\right)=\left(\begin{array}{cccccc}
\delta^{2} / 2(V-J / 2)\left[\cos \left(q_{x}\right)+\cos \left(q_{y}\right)\right] & \delta / 2 & 0 & 0 & 0 & 0 \\
\delta / 2 & 0 & 0 & 0 & 0 & 0 \\
0 & 0 & J r^{2} & 0 & 0 & 0 \\
0 & 0 & 0 & J r^{2} & 0 & 0 \\
0 & 0 & 0 & 0 & J r^{2} & 0 \\
0 & 0 & 0 & 0 & 0 & J r^{2}
\end{array}\right)^{-1}
$$

and

$$
\Pi_{a b}\left(\mathbf{q}, i \nu_{n}\right)=-\frac{1}{N_{s}} \sum_{\mathbf{k}} h_{a}\left(\mathbf{k}, \mathbf{q}, \epsilon_{\mathbf{k}}-\epsilon_{\mathbf{k}-\mathbf{q}}\right) h_{b}\left(\mathbf{k}, \mathbf{q}, \epsilon_{\mathbf{k}}-\epsilon_{\mathbf{k}-\mathbf{q}}\right) g\left(\mathbf{k}, \mathbf{q}, i \nu_{n}\right)-\delta_{a}^{R} \delta_{b}^{R} \frac{1}{N_{s}} \sum_{\mathbf{k}} \frac{\epsilon_{\mathbf{k}-\mathbf{q}}-\epsilon_{\mathbf{k}}}{2} n_{F}\left(\epsilon_{\mathbf{k}}\right),
$$

with

$$
g\left(\mathbf{k}, \mathbf{q}, i \nu_{n}\right)=\frac{\left[n_{F}\left(\epsilon_{\mathbf{k}-\mathbf{q}}\right)-n_{F}\left(\epsilon_{\mathbf{k}}\right)\right]}{i \nu_{n}+\epsilon_{\mathbf{k}-\mathbf{q}}-\epsilon_{\mathbf{k}}},
$$

where $i \nu_{n}$ are bosonic Matsubara frequencies.

The instability of the paramagnetic phase is indicated by the divergence of the static susceptibilities defined by $D_{a b}\left(\mathbf{q}, i \nu_{n}=0\right) \stackrel{31}{\underline{3}}$ As in Ref.[29] we study eigenvalues and eigenvectors of the $6 \times 6$ matrix $\left[D_{a b}\left(\mathbf{q}, i \nu_{n}\right)\right]^{-1}$ at $i \nu_{n}=0$. When an eigenvalue crosses zero at a given doping rate, temperature $T$ and momentum $\mathbf{q}$, then an instability occurs toward a phase characterized by the corresponding eigenvector $\boldsymbol{v}$.

\section{Flux phase instability of the paramagnetic state}

Here we choose suitable parameters for cuprates $t^{\prime} / t=$ $0.35, J / t=0.3$, where the realistic value for $t$ is around $400 \mathrm{meV}$. In what follows the lattice constant $a$ and the hopping $t$ are considered as length and energy units, respectively.

At $T=0$ the leading instability is the flux phase which occurs at $\delta=\delta_{c} \sim 0.17$ for $\mathbf{q} \sim \mathbf{Q}=(\pi, \pi)$, while at finite $T$ the instability occurs at $\mathbf{q}=\mathbf{Q}$. The flux phase instability is associated with an eigenvector $\boldsymbol{v} \sim \frac{1}{\sqrt{2}}(0,0,0,0,1,-1)$ of the $D_{a b}\left(\mathbf{q}, i \omega_{n}=0\right)$ susceptibility matrix, which leads to staggered circulating currents and a flux that penetrates the plaquette in the square lattice $\underline{27-29}$

At this point is worth to note that similar circulating currents where proposed by Varma for describing the

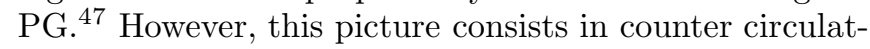
ing currents within the $\mathrm{CuO}_{2}$ unit cell and do not break the translational symmetry as the flux phase.

Below the transition temperature $T_{F P}$, the flux phase is characterized by a $2 \times 2$ Green function

$$
\widehat{G}^{-1}\left(\mathbf{k}, i \omega_{n}\right)=\left(\begin{array}{cc}
i \omega_{n}-\epsilon_{\mathbf{k}} & i \Phi \gamma(\mathbf{k}) \\
-i \Phi \gamma(\mathbf{k}) & i \omega_{n}-\epsilon_{\mathbf{k}-\mathbf{Q}}
\end{array}\right)
$$

In Eq.(11) $i \omega_{n}$ are fermionic Matsubara frequencies and $\gamma(\mathbf{k})=\frac{1}{2}\left(\cos k_{x}-\cos k_{y}\right)$, showing the $d$-wave character of the FP order parameter $\Phi$. This order parameter satisfies the following equation 


$$
\Phi=-\frac{2 J}{N_{s}} \sum_{\mathbf{k}, \omega_{n}} \frac{\Phi \gamma^{2}(\mathbf{k})}{\left(i \omega_{n}-\epsilon_{\mathbf{k}}\right)\left(i \omega_{n}-\epsilon_{\mathbf{k}-\mathbf{Q}}\right)-[\Phi \gamma(\mathbf{k})]^{2}}
$$

In Fig. 11(a) solid line shows the temperature $T_{F P}$ as a function of doping where the order parameter $\Phi$ is developed, i.e., where the FP instability takes place. $T_{F P}$ decreases when doping increases, and vanishes at a $\mathrm{QCP}$ at $\delta_{c}$. At $T=0$ the order parameter $\Phi$ decreases with doping as depicted by the solid line in Fig. 11(b). In Fig. 1(c) we show, for $\delta=0.10$, the behavior of $\Phi$ as a function of the temperature. We note here that the $t-J$ model treated in large- $\mathrm{N}$ offers a route for describing the $d$-CDW phase, where $\Phi$ and $T_{F P}$ are obtained from the microscopic treatment and not introduced as input parameters.

\section{Self-energy fluctuations above $T_{F P}$}

Since $D_{a b}$ enters explicitly in the self-energy expression [Eq.(5)], $\Sigma(\mathbf{k}, \omega)$ probes the proximity to the flux instability. At dopings and temperatures near the FP instability, the most important contribution to the selfenergy can be obtained after projecting $\Sigma(k, \omega)$ on the FP eigenvector $\boldsymbol{v}$. The obtained self-energy is 34,35

$$
\begin{aligned}
\operatorname{Im} \Sigma_{f l u x}(\mathbf{k}, \omega) & =-\frac{J^{2}}{N_{s}} \sum_{\mathbf{q}} \Gamma^{2}(\mathbf{q}, \mathbf{k}) \operatorname{Im} \chi_{f l u x}\left(\mathbf{q}, \omega-\epsilon_{\mathbf{k}-\mathbf{q}}\right) \\
& \times\left[n_{F}\left(-\epsilon_{\mathbf{k}-\mathbf{q}}\right)+n_{B}\left(\omega-\epsilon_{\mathbf{k}-\mathbf{q}}\right)\right]
\end{aligned}
$$

which shows the explicit contribution of the flux susceptibility

$$
\chi_{f l u x}(\mathbf{q}, \nu)=\left[2 J r^{2}-\Pi(\mathbf{q}, \nu)\right]^{-1} .
$$

In the above expression $\Pi\left(\mathbf{q}, i \nu_{n}\right)$ is

$$
\Pi\left(\mathbf{q}, i \nu_{n}\right)=-\frac{J^{2}}{N_{s}} \sum_{\mathbf{k}} \Gamma^{2}(\mathbf{q}, \mathbf{k}) \frac{\left[n_{F}\left(\epsilon_{\mathbf{k}-\mathbf{q}}\right)-n_{F}\left(\epsilon_{\mathbf{k}}\right)\right]}{i \nu_{n}+\epsilon_{\mathbf{k}-\mathbf{q}}-\epsilon_{\mathbf{k}}}
$$

where $\Gamma(\mathbf{q}, \mathbf{k})=r\left[\sin \left(k_{x}-q_{x} / 2\right)-\sin \left(k_{y}-q_{y} / 2\right)\right]$. Since the instability takes place at $\mathbf{q}=\mathbf{Q}, \Gamma(\mathbf{Q}, \mathbf{k})$ is then proportional to $\gamma(\mathbf{k})$, as expected due to the $d$-wave character of the FP instability. It can be shown that the onset temperature $T_{F P}$, where the flux instability takes place [Fig. 1 (a)], is the same temperature where the static $\left(i \nu_{n}=0\right)$ flux susceptibility $\chi_{\text {flux }}$ diverges at $\mathbf{q}=\mathbf{Q}$ At $T \rightarrow T_{F P}$ a soft mode $\left[\operatorname{Im} \chi_{\text {flux }}(\mathbf{Q}, \nu)\right]$ reaches $\nu=0$ freezing the FP ${ }^{34}$ Inset of Fig. 11(a) shows the softening of this mode approaching $T_{F P}$ from above for $\delta=0.10$. As shown below, the mode associated with the FP instability plays an important role in $\Sigma_{f l u x}(\mathbf{k}, \omega)$ at low doping.
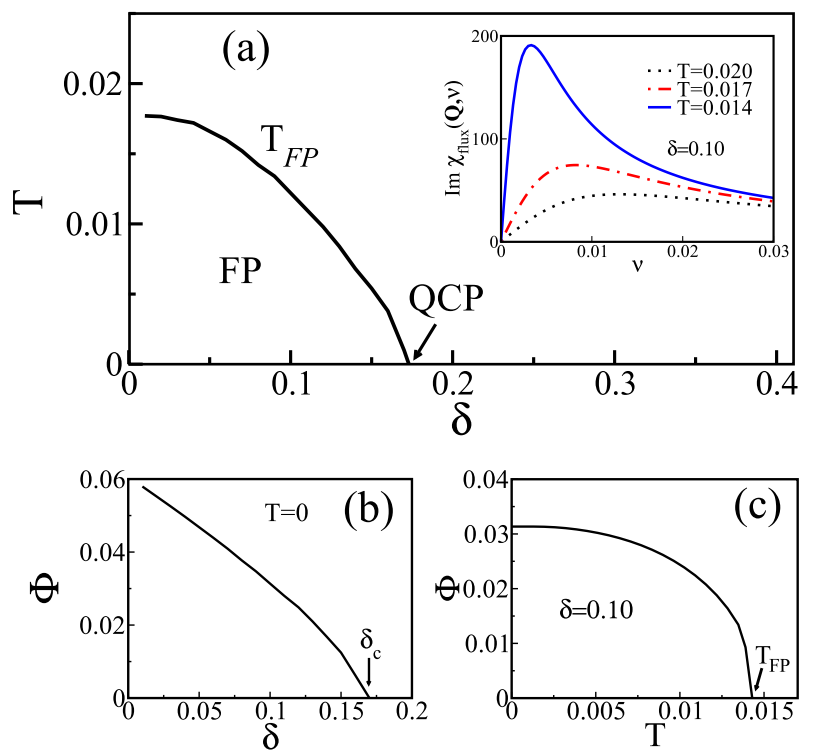

FIG. 1: (Color online) (a) $T_{F P}$ as a function of doping. At $T_{F P}$ the Fermi liquid state becomes unstable against a flux phase or $d$-CDW. The inset shows the softening of the $d$-wave flux mode approaching $T_{F P}$ for $\delta=0.10$. (b) Flux phase order parameter $\Phi$ as a function of doping for $T=0$. (c) $\Phi$ versus temperature for $\delta=0.10 . \eta=0.02$ was used in the analytical continuation $i \nu_{n} \rightarrow \nu+i \eta$.

\section{PSEUDOGAP SELF-ENERGY EFFECTS: COMPARISON WITH ARPES AND ADMR EXPERIMENTS}

In the FP state, below $T_{F P}$, the one-particle Green function [i.e., element $(1,1)$ from Eq.(11)] is

$$
G\left(\mathbf{k}, i \omega_{n}\right)=\frac{1}{i \omega_{n}-\epsilon_{\mathbf{k}}-\Sigma_{F P}\left(\mathbf{k}, \omega_{n}\right)}
$$

where

$$
\Sigma_{F P}\left(\mathbf{k}, i \omega_{n}\right)=\frac{[\Phi \gamma(\mathbf{k})]^{2}}{i \omega_{n}-\epsilon_{\mathbf{k}-\mathbf{Q}}}
$$

At this point it is important to remark the difference between $\Sigma_{F P}\left[\right.$ Eq.(17)] and $\Sigma_{\text {flux }}[$ Eq.(13)]. While the former is an effective self-energy that describes the broken symmetry state (see also Ref.[3] ), the second one represents dynamical self-energy effects in the paramagnetic state.

The Green function (16) leads to a FS that consists of four hole pockets around nodal direction with low spectral weight in the outer part of the pockets ${ }^{48}$ [see Fig. 2(a)]. However, most of the ARPES reports show disconnected arcs ${ }^{13,49-61}$ and to a lesser extent show pockets,, $62-67$ without full agreement on shape and localization of the pockets inside the Brillouin zone. Since experiments do not show clearly any turn at the end of 


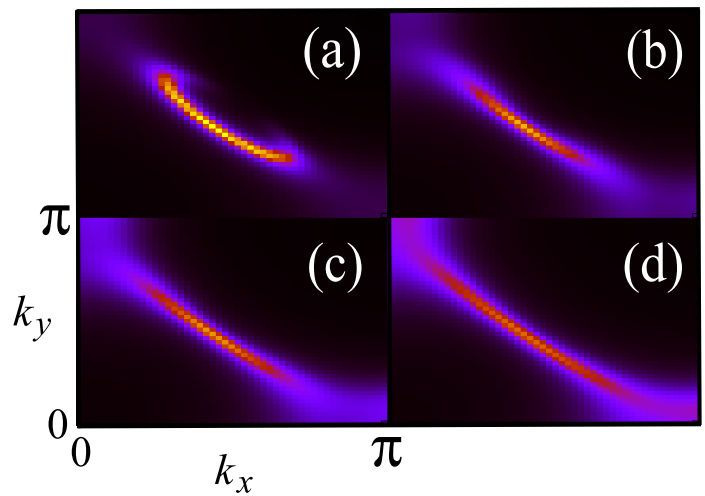

FIG. 2: (Color online) Short-ranged flux phase results. Panels (a)-(d) show results for the spectral intensity at $\omega=0$ vs $k_{x}, k_{y}$ for $\delta=0.07$ for $\xi \rightarrow \infty, \xi=5, \xi=2$ and $\xi=1$ respectively. For $\xi \rightarrow \infty$ [panel (a)] pockets predicted by the long-ranged FP case are obtained, while for the short-ranged case [panels (b)-(d)] the pockets wash out resembling an arc.

the arc, the existence of a pocket and the breaking of translational symmetry is doubtful. $\underline{3}$ Furthermore, there are several drawbacks to be addressed. FP, as well as $d$-CDW, predicts well defined QP peaks everywhere on the Brillouin zone while most of the ARPES show coherent peaks only near the nodal direction, and near the antinode the QP peak is always broad. $\stackrel{49,68}{ }$ On the other hand, in the FP scenario a true phase transition takes place at $T_{F P}$ where a true gap $\Phi$ is developed. However, experiments show a smooth crossover in the temperature behavior of normal state properties instead of an abrupt transition. 51,52,69 Consistent with this observation, ARPES shows that the PG is filling up but not closing with increasing $T$, giving the impression of a smooth crossover of the spectral properties ${ }^{50}-52$

These points can be discussed phenomenologically by assuming a short-ranged FP (or $d$-CDW) scenario 70 In this context the self-energy can be written as

$$
\Sigma_{F P}^{(S R)}\left(\mathbf{k}, i \omega_{n}\right)=\sum_{\mathbf{q}} \frac{P(\mathbf{q})[\Phi \gamma(\mathbf{k}-\mathbf{q})]^{2}}{i \omega_{n}-\epsilon_{\mathbf{k}-\mathbf{q}}}
$$

where $P(\mathbf{q})$ represents a Lorentzian distribution of ordering wave vector $\mathbf{Q}$ with a width $1 / \xi$ associated with a finite correlation length $\xi$.

Using the obtained $\Phi$ of Fig. 11(b), we show in Fig. $2(\mathrm{a})$-(d) the underlying FS for $\xi \rightarrow \infty, \xi=5, \xi=2$ and $\xi=1$ respectively, for $\delta=0.07$. While for $\xi \rightarrow \infty$ pockets are obtained, with decreasing $\xi$ the pockets wash out resembling an arc. In addition, the length of the arcs increases with decreasing $\xi$.

Although the short-ranged scenario seems to be better than the long-ranged case for describing ARPES, there are still several drawbacks: (a) The doping and temperature dependence for the correlation length $\xi$ and $\Phi$ is required phenomenologically in order to fit the doping and temperature dependence of ARPES features. (b) The presence of a finite $\Phi$ means also that a true phase transition exists at a certain temperature $T^{*} \equiv T_{F P}>T_{c}$, below which a true gap $\Phi$ emerges. As discussed above, this point is not clear from an experimental point of view. (c) The full Fermi surface is recovered either if $T>T_{F P}$ $(\Phi=0)$ or when the correlation length is lower than the lattice constant $a(\xi \ll 1)$. In both latter cases, the resulting spectral function is isotropic along the FS. As discussed above, ARPES experiments show that well defined QP peaks are observed only near the nodal direction, while near the antinodal direction the QP peaks are always broad, 49,68 i.e., the shape of the spectral function is very anisotropic on the FS even for $T>T^{*}$. (d) Finally, the $\omega$ dependence of $\Sigma_{F P}^{(S R)}$ is inherited only from the $\omega$ dependence of the bare Green function [Eq.(18)], so that dynamical properties as transport cannot be discussed in this context.

Next we will show that the presence of $\Sigma_{\text {flux }}$ in the paramagnetic state near the instability accounts for several observed features, without assuming additional phenomenological parameters or approximations beyond the large- $\mathrm{N}$ approximation.

As discussed in Sec. II, upon approaching $T_{F P}$ from above $\operatorname{Im} \chi_{\text {flux }}(\mathbf{Q}, \omega)$ develops a soft energy mode [inset of Fig. [1(a)] at low doping and near $T_{F P}$. Thus, contributions from $\Sigma_{\text {flux }}$ are the leading ones at low doping and low energy. In addition, they are strongly dependent on $J$ and doping, 31 and have $d$-wave anisotropy. Figure 3 shows $\operatorname{Im} \Sigma_{\text {flux }}$ at the Fermi momenta $\mathbf{k}_{\mathbf{F}}^{\mathbf{N}}$ (nodal) and $\mathbf{k}_{\mathbf{F}}^{\mathbf{A N}}$ (antinodal) for $T=0.01$, for $\delta=0.14$ [panel (a)] and $\delta=0.26$ [panel (b)]. At $\mathbf{k}_{\mathbf{F}}^{\mathbf{N}} \operatorname{Im} \Sigma_{\text {flux }}$ is weak and leads to a well defined QP peak in the nodal direction for all doping. ${ }^{34-36}$ However, the behavior at $\mathbf{k}_{\mathbf{F}}^{\mathbf{A N}}$ is very peculiar at low doping. Instead of a maximum at $\omega=0, \operatorname{Im} \Sigma_{\text {flux }}$ shows a minimum in the form of a dip. This behavior, which is in contrast to the expected one for a Fermi-liquid, $\stackrel{71,72}{ }$ is the main reason for the $\mathrm{PG}$ [Fig. 3(e)] and Fermi arc (FA) formation [Fig. 3(c) for $\delta=0.14]$. With increasing doping and temperature the dip at $\omega \sim 0$ washes out, and $\operatorname{Im} \Sigma_{\text {flux }}$ develops the expected maximum at $\omega=0$ [Fig. 33(b)], leading to the expected large hole FS [Fig. 33(d) for $\delta=0.26$ ]. In addition, the leading edge of the PG near the antinode fills up with temperature as in the experiments [Fig. 3(e)] ${ }^{34-36}$ From the above discussion, we use also the name PG selfenergy for $\Sigma_{\text {flux }}$. As already discussed in Ref.[37], our approach offers a microscopic theory for understanding the phenomenological short-ranged order approach and may describe recent ARPES experiments. ${ }^{13}$

In Fig. 4(a) we show the QP weight $Z_{\text {flux }}=(1-$ $\left.\left.\frac{\partial \operatorname{Re} \Sigma_{\text {flux }}}{\partial \omega}\right|_{\omega=0}\right)^{-1}$ at $T=0$ for the FS points at the node (double dotted dashed line) and the antinode (solid line). The difference between $Z_{\text {flux }}^{A N}$ and $Z_{\text {flux }}^{N}$ shows the large anisotropy of $\Sigma_{\text {flux }}$ on the FS. Besides, $Z_{\text {flux }}^{A N}$ vanishes at the QCP 35

In contrast to $\Sigma_{F P}^{(S R)}, \Sigma_{\text {flux }}$ can be used for discussing 

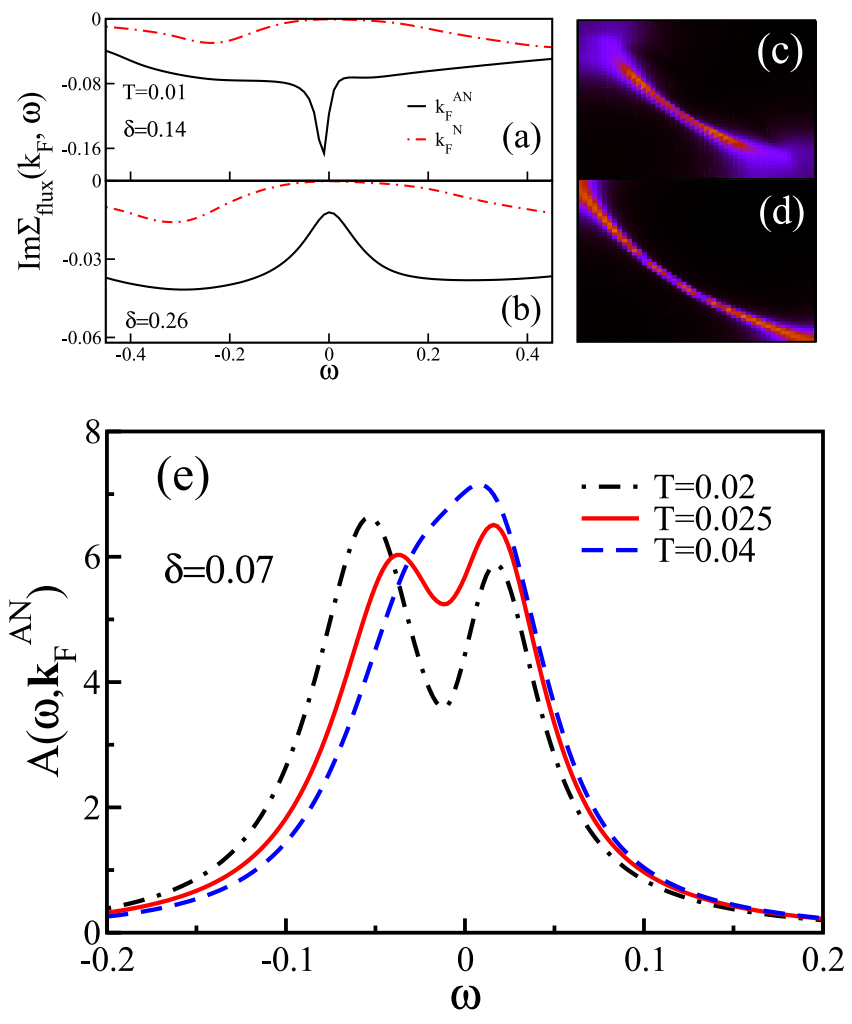

FIG. 3: (Color online) (a) and (b) imaginary part of the selfenergy for the nodal (dotted dashed line) and the antinodal (solid line) $\mathbf{k}_{\mathbf{F}}$ for doping $\delta=0.14$ and $\delta=0.26$ respectively, at $T=0.01$. (c) and (d) underlying FS for $\delta=0.14$ and $\delta=$ 0.26 respectively. While for $\delta=0.26$ the expected large hole FS is obtained, for $\delta=0.14$ the unusual dips near the antinode presented by the self-energy [panel (a)] develops an arc. (e) spectral function obtained with $\Sigma_{\text {flux }}$ at $\delta=0.07$ for several temperatures. A PG-like feature is developed at temperatures near but above $T_{F P}$, and fills with increasing temperature, in agreement with that observed in ARPES experiments.

transport. $\Sigma_{\text {flux }}$ leads to an anisotropic scattering rate on the FS, i.e., $1 / \tau_{\text {flux }}\left(\mathbf{k}_{\mathbf{F}}\right) \equiv-2 \operatorname{Im} \Sigma_{\text {flux }}\left(\mathbf{k}_{\mathbf{F}}, \omega=\right.$ $0)$. As recently suggested, ${ }^{33} 1 / \tau_{\text {flux }}\left(\mathbf{k}_{\mathbf{F}}\right)$ possesses an anisotropy on the FS close to $d$-wave [Fig. 4 panel (b)], and vanishes in OD [panel (c) for the average in the FS $\left.\left\langle 1 / \tau_{\text {flux }}\right\rangle_{F S}\right]$. As discussed in ADMR experiments these are the main characteristics for one of the two observed scattering rates, $73-75$

The background color in Fig. [5 shows, in the $T-\delta$ plane, the spectral function intensity obtained with $\Sigma_{\text {flux }}$, at $\omega=0$ for the antinodal $\mathbf{k}_{\mathbf{F}}$. This figure shows a crossover (double dashed dotted line) from a region of low intensity at low doping and high temperature to a region of high intensity at large doping and low temperature 35 As in Ref. [76] we identify the former region with an incoherent metal where the QP peak is broad, whereas in the latter region the QP is well defined as expected for a coherent metal. Note that the coherent to incoherent crossover, and its relation with the $\mathrm{PG}$, can not be discussed in the
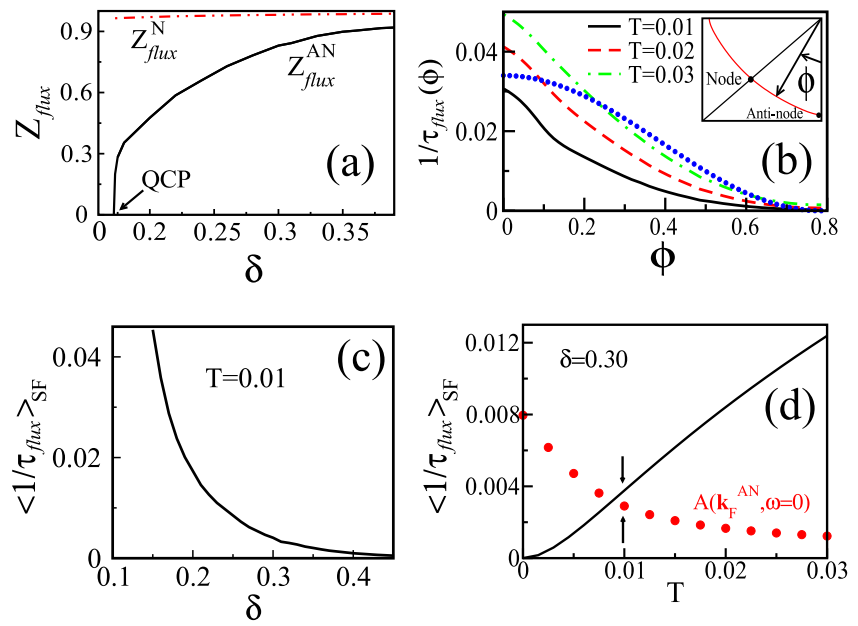

FIG. 4: (Color online) (a) QP weight $Z_{\text {flux }}$ at $T=0$ as a function of doping, for the nodal $\left(Z_{\text {flux }}^{N}\right)$ and antinodal $\left(Z_{\text {flux }}^{A N}\right)$ momentum on the FS. The QP weight is strongly anisotropic on the FS, and vanishes at the antinode upon approaching the QCP. (b) anisotropic scattering rate $1 / \tau_{\text {flux }}\left(\mathbf{k}_{\mathbf{F}}\right)=$ $-2 \operatorname{Im} \Sigma_{\text {flux }}\left(\mathbf{k}_{\mathbf{F}}, \omega=0\right)$, on the FS for $\delta=0.20$ and several temperatures as a function of the FS angle $\phi$ running from the antinode $\phi=0$ to the node $\phi=\pi / 4$ (shown in the inset). $1 / \tau_{\text {flux }}$ follows approximately a $d$-wave shape (dotted line). (c) average on the FS of $1 / \tau_{\text {flux }}$ at $T=0.01$ as a function of doping, showing that $\left\langle 1 / \tau_{\text {flux }}\right\rangle_{F S}$ is strongly doping dependent and vanishes in OD. (d) average on the FS of $1 / \tau_{\text {flux }}$ (solid line) and the corresponding spectral function intensity (dots) at $\mathbf{k}_{\mathbf{F}}^{\mathbf{A N}}$ and $\omega=0$, for $\delta=0.30$ as a function of temperature. Below a certain temperature (black arrows), the spectral function intensity at $\omega=0$ increases with decreasing $T$ faster than above that temperature. The figure shows that this temperature is close to that where a change in the curvature of $\left\langle 1 / \tau_{\text {flux }}\right\rangle_{F S}$ vs $T$ occurs.

context of the phenomenological short-ranged scenario.

Similar to Ref. [76], above the crossover temperature (which increases with doping) the intensity of the spectral function decreases with $T$ slower than below the crossover temperature. This crossover is also linked to the behavior of the anisotropic scattering rate. In Fig. 4(d) we show with black arrows that the spectral intensity (dots) increases faster with decreasing temperature at approximately the same temperature where a change in the curvature of $\left\langle 1 / \tau_{\text {flux }}\right\rangle_{F S}$ (solid line) occurs. In Refs. 76, 77. this crossover was also identified with a change in the temperature behavior of the resistivity. In Ref. [47] the incoherent-coherent crossover was also discussed in terms of the paramagnetic state and the QCP scenario.

Dashed line in Fig. $5\left(T_{\text {arcs }}\right)$ shows a crossover temperature above which the large hole FS is recovered. Thus, FAs occur between $T_{F P}$ and $T_{\text {arcs }}$ in the paramagnetic phase and without the breaking of translational symmetry. Although dashed line ends at the QCP, $T_{\text {arcs }}$ is not a critical transition temperature. Interestingly, $T_{\text {arcs }}$ is close to $T^{u p}$ obtained in reference [33] which is defined 


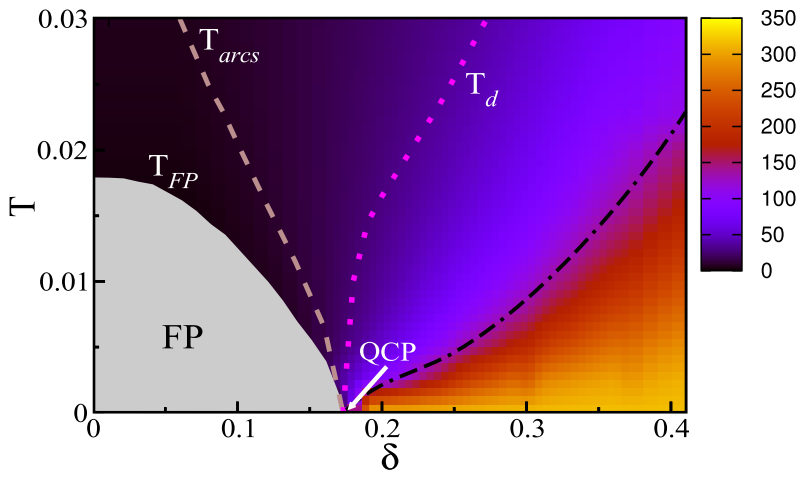

FIG. 5: (Color online) Temperature versus doping phase diagram without superconductivity. In the gray area below $T_{F P}$ the translational symmetry is broken and pockets are obtained. $T_{\text {arcs }}$ (dashed line) shows the crossover temperature below which the spectral functions have a pseudogap near the antinode and Fermi arcs are developed. Therefore, we can associate $T_{\text {arcs }}$ with $T^{*}$. Dotted line $\left(T_{d}\right)$ is the temperature above which the self-energy presents a dip in $\omega=0$. The background color shows the antinodal spectral function intensity at $\omega=0$. Double dashed dotted line shows the crossover from a bad metal at low doping and high temperature to a good metal at high doping and low temperature.

as the temperature where $\left\langle 1 / \tau_{\text {flux }}\right\rangle_{F S}$ shows an upturn, allowing us to relate the onset of the pseudogap phase observed in ARPES with that obtained by resistivity experiments $\frac{78}{78}$ Thus, we associate $T_{\text {arcs }}$ with $T^{*}$.

Although arcs wash out above $T_{\text {arcs }}$, the self-energy does not behaves as expected from a Fermi liquid (FL). Between $T_{\text {arcs }}$ and $T_{d}$ (dotted line), $\Sigma_{\text {flux }}$ shows a dip near the antinode, and only below $T_{d}$ the expected maximum at $\omega=0$ for the whole FS is obtained. The fact that there are no arcs between $T_{\text {arcs }}$ and $T_{d}$ can be explained as follows. For the existence of arcs $\Sigma_{\text {flux }}$ must show a dip at $\omega \sim 0$ [see Fig. 3(a)], but in addition, the height of the dip must be larger than its half-width. See Ref.[79] where this point was discussed in the context of the Pomeranchuk instability where broad spectral functions on the FS and no PG were obtained, but the self-energy is not FL-like. Thus, between $T_{\text {arcs }}$ and $T_{d}$ while the full FS is recovered, the shape of the spectral functions is very anisotropic on the FS, i.e., QP peaks are broad near the antinode and sharp near the node. Such anisotropy is lost with further increasing doping.

In summary, the fluctuating FP approach has several advantages over the short-ranged case: (a) The $T_{F P}$ line is defined from microscopic treatment and depends only on the expression of $\chi_{\text {flux }}$, which on the other hand enters in $\Sigma_{\text {flux }}$. In other words, in the fluctuating PG framework there are no additional assumptions or input parameters beyond our microscopic treatment of the $t-J$ model. (b) There is no need for the occurrence of a true phase transition, since arcs are originated dynamically due to the interaction between carriers and FP fluctuations at $T>T_{F P}$, implying that long-range order is not broken. (c) In the region between $T_{\text {arcs }}$ and $T_{d}$, although the full FS is recovered, it can be shown (see Fig. 12(b) of Ref. [36]) that the spectral function shows well defined QP peaks near the nodal direction and broad QP peaks near the antinodal direction, as observed in ARPES experiments. (d) $\Sigma_{\text {flux }}$ contains its own frequency and temperature dependence which, as mentioned above, allows to discuss transport. Finally, we remark that for a more quantitative agreement with the phase diagram of cuprates, $T_{F P}$ should be located at lower temperatures. See next section for further discussion.

Cluster dynamical mean-field theory in Ref. [80] shows that the spectral functions develop a PG and FAs which change with doping and temperature in close agreement with our results. In addition, the PG and FAs formation were assigned to a self-energy behavior in which the imaginary part near $\omega=0$ shows a dip instead of a maximum. The agreement between our results and cluster dynamical mean-field theory is satisfactory. Although our approach is not exact, it offers a microscopic mechanism which usually is less evident from purely numerical methods.

In the next section we discuss the interplay between the PG and superconductivity.

\section{SUPERCONDUCTIVITY}

The large- $\mathrm{N}$ treatment of the $t-J$ model shows a $d$ wave superconductivity which is mainly mediated by non-retarded interactions $J\left(\mathbf{k}-\mathbf{k}^{\prime}\right)=2 J\left[\cos \left(k_{x}-k_{x}^{\prime}\right)+\right.$ $\left.\cos \left(k_{y}-k_{y}^{\prime}\right)\right] \stackrel{28}{\underline{30}}$ The gap equation for the $d$-wave superconducting order parameter $\Delta(\mathbf{k})$ can be written as:

$$
\Delta(\mathbf{k})=-4 J \gamma(\mathbf{k}) \frac{T}{N_{s}} \sum_{\mathbf{k}^{\prime}, n^{\prime}} \gamma\left(\mathbf{k}^{\prime}\right) G_{12}^{0}\left(\mathbf{k}^{\prime}, i \omega_{n^{\prime}}\right),
$$

where $G_{12}^{0}$ is the element $(1,2)$ of the bare Green function

$$
\left(\widehat{G}^{0}\right)^{-1}\left(\mathbf{k}, i \omega_{n}\right)=\left(\begin{array}{cc}
i \omega_{n}-\epsilon_{\mathbf{k}} & -\Delta(\mathbf{k}) \\
-\Delta(\mathbf{k}) & i \omega_{n}+\epsilon_{\mathbf{k}}
\end{array}\right) .
$$

Figure 6 shows that the bare superconducting critical temperature, called $T_{c}^{\text {bare }}$ (dotted dashed line), increases monotonically with decreasing doping. The term bare is used here because the gap equation (19) does not contain self-energy effects. We note that the mean-field superconducting gap equation (19) is identical to Eq. (12) for the FP order parameter $\Phi$ if $\epsilon_{\mathbf{k}-\mathbf{Q}}$ is replaced by $-\epsilon_{\mathbf{k}}$ in (12).

Taking into account self-energy effects, the gap equation results:

$$
\Delta(\mathbf{k})=-4 J \gamma(\mathbf{k}) \frac{T}{N_{s}} \sum_{\mathbf{k}^{\prime}, n^{\prime}} \gamma\left(\mathbf{k}^{\prime}\right) G_{12}\left(\mathbf{k}^{\prime}, i \omega_{n^{\prime}}\right) .
$$

In Eq. (21) the renormalized Green function $\widehat{G}$ is: $\unrhd^{81}$ 


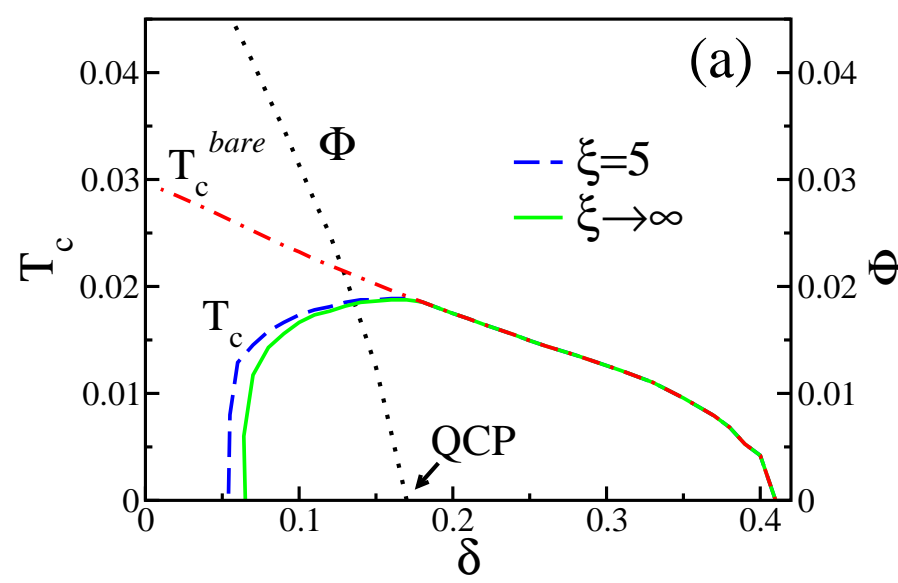

FIG. 6: (Color online) Results of short-ranged FP for $T_{c}$. Dotted line shows the gap $\Phi$ versus doping [same as Fig. 1(b)]. Dotted dashed line shows $T_{c}^{\text {bare }}$ obtained from Eq. (19) without self-energies effects. Solid line and dashed line are the results for $T_{c}$ obtained assuming in $\Sigma_{F P}^{(S R)}$ [Eq.(18)] a correlation length $\xi \rightarrow \infty$ and $\xi=5$, respectively. For $\delta>\delta_{c}, \Phi=0$, and therefore $\Sigma_{F P}^{(S R)}=0$, thus $T_{c}=T_{c}^{\text {bare }}$.

$$
\widehat{G}^{-1}\left(\mathbf{k}, i \omega_{n}\right)=\left(\widehat{G}^{0}\right)^{-1}\left(\mathbf{k}, i \omega_{n}\right)-\widehat{\Sigma}\left(\mathbf{k}, i \omega_{n}\right)
$$

where

$$
\widehat{\Sigma}\left(\mathbf{k}, i \omega_{n}\right)=\left(\begin{array}{cc}
\Sigma\left(\mathbf{k}, i \omega_{n}\right) & 0 \\
0 & -\Sigma\left(\mathbf{k},-i \omega_{n}\right)
\end{array}\right) .
$$

Eq.(21) means that we assume that excitations entering in $\Sigma$ do not affect the non-retarded pairing.

\section{Short-ranged flux phase case}

Figure 6 shows results for $T_{c}$ obtained using the selfenergy $\Sigma_{F P}^{(S R)}$ (18) in (23). For the long-ranged case $\xi \rightarrow \infty$ (solid line), results are in close agreement with the treatment of Ref. 28], where the competition between $\Phi$ and $\Delta$ was considered self-consistently. Interestingly, even for short correlation length of about $\xi=5$, we obtain a well defined DSB for $T_{c}$ (dashed line). Since for $\delta>\delta_{c}, \Phi=0\left(\Sigma_{F P}^{(S R)}=0\right)$, then $T_{c}$ follows $T_{c}^{\text {bare }}$ (dotted dashed line).

However, as discussed in the previous section, the FP fluctuating approach has several advantages over the short-ranged $d$-CDW. Thus, it is interesting to see if fluctuations near the FP instability can lead to a DSB for $T_{c}$.

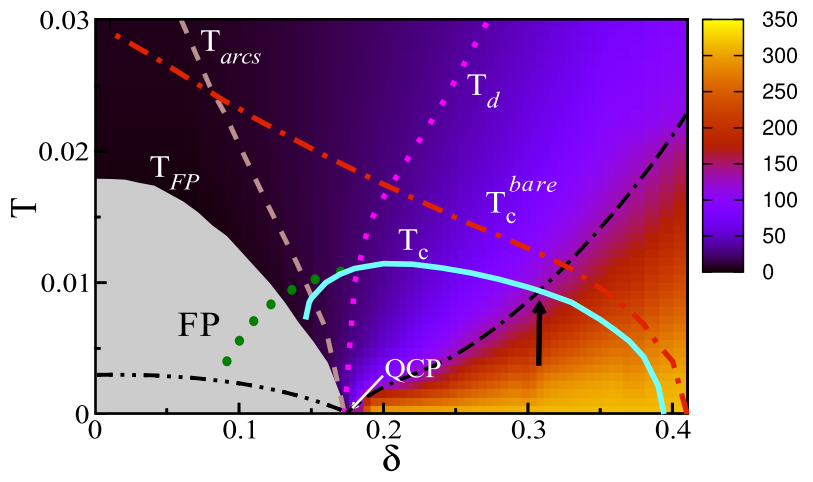

FIG. 7: (Color online) Temperature versus doping phase diagram with the inclusion of superconductivity. Dotted dashed, dashed and dotted line shows $T_{c}^{\text {bare }}$ (same as Fig[6), $T_{\text {arcs }}$ and $T_{d}$ respectively. Solid line shows the dome-shaped behavior of $T_{c}$ obtained in presence of $\Sigma_{\text {flux }}$. Interestingly, $T_{c}$ go first parallel to $T_{c}^{\text {bare }}$, and shows a downward deviation (black arrow) at the crossover temperature from a coherent to an incoherent metal. Double dotted dashed line illustrates a situation where the FP instability occurs at lower temperature. The expected superconducting temperature for this situation is illustrated by the dots next to $T_{c}$ (see text for discussion).

\section{Role of the fluctuating pseudogap self-energy $\Sigma_{f l u x}$ on superconductivity}

The role of the PG self-energy on the doping behavior of $T_{c}$ can be studied by the gap equation (21) considering $\Sigma_{\text {flux }}[\mathrm{Eq}$.(13)]. In our view the PG fluctuations do not glue the electrons into Cooper pairs.

Since we are interested in the doping behavior of $T_{c}$, it is not necessary to consider the effects of the superconducting gap on $\Sigma_{\text {flux }}$ which, then, can be calculated in the normal state.

We remark that $d$-wave superconductivity ${ }^{82}$ and a $\mathrm{DSB}^{83,84}$ for $T_{c}$ in the $t-J$ model were supported by numerical studies.

Figure 7 shows that $T_{c}$ (solid line) moves at first parallel to the results for $T_{c}^{\text {bare }}$ (dotted dashed line) with decreasing doping. For high doping and low temperature, $\Sigma_{\text {flux }}$ behaves as in a conventional Fermi-liquid, presenting a maximum at $\omega=0$ as discussed in the previous section. Then, for $\delta \gtrsim 0.3$ the reduction of $T_{c}$ from $T_{c}^{b a r e}$ is due to conventional Fermi-liquid self-energy effects where $T_{c}$ diminishes following approximately the form $\log \left(T_{c} / T_{c}^{\text {bare }}\right) \sim\left(1-Z^{-1}\right)$.

However, $T_{c}$ does not follow the same trend as $T_{c}^{\text {bare }}$ for $\delta<0.30$. $T_{c}$ deviates from that trend at about the crossover line between the coherent and the incoherent metal (see black arrow in Fig. 7). Approaching the QCP, $T_{c}$ reaches its maximum and begins to fall with decreasing doping, showing clear tendencies to develop a DSB. The fact that the fall of $T_{c}$ occurs approaching the QCP allows to associate this behavior to the opening of the fluctuating PG, showing the relevance of critical fluctua- 
tions near the instability. This fact shows that the DSB of $T_{c}$ is related to the properties of the incoherent metal near the instability, and not to the coherent metal at low temperature and large doping. It is important also to remark that the coherent metal properties predict that $T_{c}$ would vanishes at the QCP where $Z \rightarrow 0$ [Fig. 4(a)]. However, $T_{c}$ tends to show a DSB for dopings lower than the QCP which indicates also the relevance of critical fluctuations in the behavior of $T_{c}$.

In a recent cluster dynamical mean-field calculation ${ }^{85}$ it was also discussed that the maximum of the superconducting transition temperature occurs near the onset of the PG.

Note that we stop the calculation of $T_{c}$ at the onset of $T_{F P}$, i.e., we do not enter inside the FP instability. For a more quantitative agreement with the phenomenology $T_{F P}$ should be at lower temperatures, e.g. following double dotted dashed line sketched in Fig. 7. Under this condition the expected trend for the superconducting critical temperature is depicted by dots. It is worth to mention that $T_{F P}$ is a mean-field critical temperature and should be interpreted as a temperature scale where the corresponding charge order, at least its fluctuation effect, may become important. It is known that the effect of fluctuations may considerably reduce the transition temperature with respect to the mean-field temperature ${ }^{86}$

In summary, the pseudogap fluctuations in the proximity to $T_{F P}$ may lead to a DSB for $T_{c}$ and, in addition, develop arcs in the paramagnetic phase which change with doping and temperature as seen in the experiments. In other words, for the existence of FAs in the normal state it is not necessary that $T<T_{F P}$ because arcs are generated by fluctuations in the proximity to the FP instability and, that fluctuations seem to be enough for triggering a DSB for $T_{c}$. The phase diagram of Fig. 7 shows close analogies with the phase diagram observed in hole doped cuprates (see for example Fig. 5 of Ref. [76] and Fig. 1 of Ref.[77]). Indeed, it has a crossover between a coherent metal and an incoherent metal, a temperature $T^{\text {arcs }}$ which can be associated with the PG temperature $T^{*}$, and tendencies to a DSB for the critical temperature $T_{c}$.

\section{DISCUSSION AND CONCLUSION}

In the context of the $t-J$ model we have discussed the dome-shaped behavior of $T_{c}$ and its relation with the pseudogap and normal state properties. The $t$ - $J$ model shows non-retarded interactions $J\left(\mathbf{k}-\mathbf{k}^{\prime}\right)$ which lead to $d$-wave superconductivity where $T_{c}$ increases monotonically with decreasing doping. Differently to conventional superconductivity, where pairing excitations can also be seen in the normal state self-energy effects, non-retarded pairing does not contribute to transport or other normal state property. However, self-energy effects may affect the doping behavior of $T_{c}$. We have shown that fluctuations near the flux phase (or $d$-CDW) instability, existing at low doping and low temperature in the $t-J$ model, lead to self-energy effects which may show a dome-shaped behavior for $T_{c}$. Moreover, (a) these self-energy effects may also describe the arc-physics observed in ARPES above $T_{c}$ without invoking a true phase transition and without translational symmetry breaking; (b) these self-energy effects are also supported by the existence of an anisotropic scattering rate observed in ADMR experiments.

A final remark. There are several experimental indications about the existence of an isotropic self-energy contribution with a high-energy scale, i.e., with properties different to those related to the PG. These experimental indications came from transport, 87 ADMR,, $73-75$ ARPES, ${ }^{88-94}$ optical conductivity, ${ }^{95}$ and Raman. ${ }^{96}$ Theoretical reports also support the existence of self-energy contributions with properties which are different to the PG ${ }^{33,38,97,98}$ If only a single universal physics is mainly involved in the DSB, the role of isotropic self-energy effects must be discussed, because a strong doping dependence of this self-energy, mainly in UD, may mask the effects of the PG in the DSB for $T_{c}$.

\section{Acknowledgments}

The authors thank to P. Horsch, J. Kokalj, R.H. McKenzie, J. Riera, H. Yamase, and R. Zeyher for valuable discussions, and to $\mathrm{H}$. Parent for critical reading of the manuscript.
1 T. Timusk and B. Statt, Rep. Prog. Phys. 62, 61 (1999).

2 V. J. Emery and S. A. Kivelson, Nature 374, 434 (1995).

3 M.R. Norman, A. Kanigel, M. Randeria, U. Chatterjee, and J. C. Campuzano, Phys. Rev. B 76, 174501 (2007).

4 C. Chien, Y. He, Q. Chen, and K. Levin, Phys. Rev. B 79, 214527 (2009)

${ }^{5}$ I. M. Vishik, W. S. Lee, R.-H. He, M. Hashimoto, Z. Hussain, T. P. Devereaux, and Z.-X. Shen, New J. Phys. 12, 105008 (2010).

6 T. Kondo, Y. Hamaya, A. D. Palczewski, T. Takeuchi, J. S. Wen, Z. J. Xu, G. Gu, J. Schmalian, and A. Kaminski, Nat. Phys. 7, 21 (2011).

7 T. Yoshida, M. Hashimoto, I. M. Vishik, Z.-X. Shen, and A. Fujimori, J. Phys. Soc. Jpn. 81, 011006 (2012).

8 S. Chakravarty, R. B. Laughlin, D.K. Morr, and C. Nayak,
Phys. Rev. B 63, 094503 (2001).

${ }^{9}$ H. Yamase, Phys. Rev. B 79, 052501 (2009).

10 C. Castellani, C. Di Castro, and M. Grilli, Phys. Rev. Lett. 75, 4650 (1995).

11 F. Becca, M. Tarquini, M. Grilli, and C. Di Castro, Phys. Rev. B 54, 12443 (1996).

12 R.-H. He, M. Hashimoto, H. Karapetyan, J. D. Koralek, J. P. Hinton, J. P. Testaud, V. Nathan, Y. Yoshida, Hong Yao, K. Tanaka, W. Meevasana, R. G. Moore, D. H. Lu, S.-K. Mo, M. Ishikado, H. Eisaki, Z. Hussain, T. P. Devereaux, S. A. Kivelson, J. Orenstein, A. Kapitulnik, Z.-X. Shen, Science 311, 1579 (2011).

13 M. Hashimoto, Rui-Hua He, K. Tanaka, J.-P. Testaud, W. Meevasana, R. G. Moore, D. Lu, H. Yao, Y. Yoshida, H. Eisaki, T. P. Devereaux, Z. Hussain and Z.-X. Shen, Na- 
ture Phys. 6, 414 (2010).

14 S. A. Kivelson, I. P. Bindloss, E. Fradkin, V. Oganesyan, J. M. Tranquada, A. Kapitulnik, and C. Howald, Rev. Mod. Phys. 75, 1201 (2003).

15 M. Vojta, Adv. Phys. 58, 699 (2009).

16 V. J. Emery and S. A. Kivelson, Physica C 209, 597 (1993).

17 K.-Y. Yang, T. M. Rice and F.-C. Zhang, Phys. Rev. B 73, 174501 (2006).

18 C. M. Varma, Phys. Rev. Lett. 83, 3538 (1999).

19 C. M. Varma, Phys. Rev. B 73, 155113 (2006).

20 T. A. Maier, D. Poilblanc, and D. J. Scalapino, Phys. Rev. Lett. 100, 237001 (2008).

21 E. van Heumen, E. Muhlethaler, A. B. Kuzmenko, H. Eisaki, W. Meevasana, M. Greven, and D. van der Marel, Phys. Rev. B 79, 184512 (2009).

22 A. Greco and R. Zeyher, Phys. Rev. B 63, 064520 (2001).

23 P.W. Anderson, Science 235, 1196 (1987).

24 P.W. Anderson, Science 316, 1705 (2007).

25 B. Mansart, J. Lorenzana, A. Mann, A. Odeh, M. Scarongella, M. Chergui, and F. Carbone, Proc. Natl. Acad. Sci. USA 110, 4539 (2013).

26 S. R. Park, Y. Cao, Q. Wang, M. Fujita, K. Yamada, S.-K. Mo, D. S. Dessau, D. Reznik, arXiv:1304.0505.

27 I. Affleck and J. B. Marston, Phys. Rev. B 37, 3774 (1988).

28 E. Cappelluti and R. Zeyher, Phys. Rev. B 59, 6475 (1999).

29 M. Bejas, A. Greco, and H. Yamase, Phys. Rev. B 86, 224509 (2012).

${ }^{30}$ R. Zeyher and A. Greco, Eur. Phys. J. B. 6, 473 (1998).

31 A. Foussats and A. Greco, Phys. Rev. B 70, 205123 (2004).

32 M. Bejas, A. Greco, and A. Foussats, Phys. Rev. B 73, 245104 (2006).

33 G. Buzon and A. Greco, Phys. Rev. B 82, 054526 (2010).

34 A. Greco, Phys. Rev. Lett. 103, 217001 (2009).

35 A. Greco, Phys. Rev. B 77, 092503 (2008).

36 M. Bejas, G. Buzon, A. Greco, and A. Foussats, Phys. Rev. B 83, 014514 (2011).

37 A. Greco and M. Bejas, Phys. Rev. B 83, 212503 (2011).

38 A. Foussats, A. Greco, and M. Bejas, Phys. Rev. B 78, 153110 (2008).

39 K. A. Chao, J. Spalek, and A. M. Oleś, J. Phys. C 10, L 271 (1977); Phys. Rev. B 18, 3453 (1978).

40 S. G. Ovschinnikov and V. V. Val'kov, Hubbard Operators in the Theory of Strongly Correlated Electrons, Imperial College Press (2004).

41 M. Brunner, F. Assaad and A. Muramatsu, Phys. Rev. B 62,15480 (2000).

42 E. Dagotto, Rev. Mod. Phys. 66, 763 (1994).

43 J. Jaklic and P. Prelovsek, Adv. Phys. 49, 1 (2000).

44 Z. Wang, Int. Journal of Modern Physics B 6, 155 (1992).

45 Z. Wang, Y. Bang and G. Kotliar, Phys. Rev. Lett 195, 2733 (1991)

46 B. S. Shastry, Phys. Rev. Lett. 107, 056403 (2011).

47 C. M. Varma, Phys. Rev. B 55, 14554 (1997).

48 S. Chakravarty, C. Nayak, and S. Tewari, Phys. Rev. B 68, 100504 (R) (2003).

49 A. Damascelli, Z. Hussain, and Z.-X.Shen, Rev. Mod. Phys. 75, 473 (2003).

50 M.R. Norman, H. Ding, M. Randeria, J. C. Campuzano, T. Yokoya, T. Takeuchi, T. Takahashi, T. Mochiku, K. Kadowaki, P. Guptasarma, and D. G. Hinks, Nature 392, 157 (1998).

51 A. Kanigel, M. R. Norman, M. Randeria, U. Chatterjee, S. Suoma, A. Kaminski, H. M. Fretwell, S. Rosenkranz, M. Shi, T. Sato, T. Takahashi, Z. Z. Li, H. Raffy, K. Kad- owaki, D. Hinks, L. Ozyuzer, and J.C. Campuzano, Nature Physics 2, 447 (2006).

52 A. Kanigel, U. Chatterjee, M. Randeria, M. R. Norman, S. Souma, M. Shi, Z. Z. Li, H. Raffy, and J. C. Campuzano, Phys. Rev. Lett. 99, 157001 (2007).

53 M. Shi, J. Chang, S. Pailhés, M. R. Norman, J. C. Campuzano, M. Månsson, T. Claesson, O. Tjernberg, A. Bendounan, L. Patthey, N. Momono, M. Oda, M. Ido, C. Mudry, and J. Mesot, Phys. Rev. Lett. 101, 047002 (2008).

${ }^{54}$ K. Terashima, H. Matsui, T. Sato, T. Takahashi, M. Kofu, and K. Hirota, Phys. Rev. Lett. 99, 017003 (2007).

55 T. Yoshida, M. Hashimoto, S. Ideta, A. Fujimori, K. Tanaka, N. Mannella, Z. Hussain, Z.-X. Shen, M. Kubota, K. Ono, S. Komiya, Y. Ando, H. Eisaki, and S. Uchida, Phys. Rev. Lett. 103, 037004 (2009).

56 T. Kondo, T. Takeuchi, A. Kaminski, S. Tsuda, and S. Shin, Phys. Rev. Lett. 98, 267004 (2007).

57 T. Kondo, R. Khasanov, T. Takeuchi, J. Schmalian, and A. Kaminski, Nature 457, 296 (2009).

${ }^{58}$ K. Tanaka, W. S. Lee, D. H. Lu, A. Fujimori, T. Fujii, Risdiana, I. Terasaki, D. J. Scalapino, T. P. Devereaux, Z. Hussain, Z.-X. Shen, Science 314, 1910 (2006).

59 W.S. Lee, I. M. Vishik, K. Tanaka, D. H. Lu, T. Sasagawa, N. Nagaosa, T. P. Devereaux, Z. Hussain, and Z.-X. Shen, Nature 450, 81 (2007).

60 J.-H. Ma Z.-H. Pan, F. C. Niestemski, M. Neupane, Y.-M. Xu, P. Richard, K. Nakayama, T. Sato, T. Takahashi, H.Q. Luo, L. Fang, H.-H. Wen, Ziqiang Wang, H. Ding, and V. Madhavan, Phys. Rev. Lett. 101, 207002 (2008).

61 Rui-Hua He, M. Hashimoto, H. Karapetyan, J. D. Koralek, J. P. Hinton, J. P. Testaud, V. Nathan, Y. Yoshida, Hong Yao, K. Tanaka, W. Meevasana, R. G. Moore, D. H. Lu, S.-K. Mo, M. Ishikado, H. Eisaki, Z. Hussain, T. P. Devereaux, S. A. Kivelson, J. Orenstein, A. Kapitulnik, and Z.-X. Shen, Science 331, 1579 (2011).

62 J. Chang, Y. Sassa, S. Guerrero, M. Månsson, M. Shi, S. Pailhés, A. Bendounan, R. Mottl, T. Claesson, O. Tjernberg, L. Patthey, M. Ido, M. Oda, N. Momono, C. Mudry, and J. Mesot, New. J. Phys. 10, 103016 (2008).

${ }^{63}$ E. Razzoli, Y. Sassa, G. Drachuck, M. Månsson, A. Keren, M. Shay, M. H. Berntsen, O. Tjernberg, M. Radovic, J. Chang, S. Pailhés, N. Momono, M. Oda, M. Ido, O. J. Lipscombe, S. M. Hayden, L. Patthey, J. Mesot, and M. Shi, New. J. Phys. 12, 125003 (2010).

64 J. Meng, G. Liu, W. Zhang, L. Zhao, H. Liu, X. Jia, D. Mu, S. Liu, X. Dong, J. Zhang, W. Lu, G. Wang, Y. Zhou, Y. Zhu, X. Wang, Z. Xu, C. Chen, and X. J. Zhou, Nature 462, 335 (2009).

${ }^{65}$ H.-B. Yang, J. D. Rameau, P. D. Johnson, T. Valla, A. Tsvelik, and G. D. Gu, Nature 456, 77 (2008).

66 K.-Y. Yang, H.-B. Yang, P. D. Johnson, T. M. Rice, and F.-C. Zhang, Euro Phys. Lett. 86, 37002 (2009).

67 H.-B. Yang, J. D. Rameau, Z.-H. Pan, G. D. Gu, P. D. Johnson, H. Claus, D. G. Hinks, and T. E. Kidd, Phys. Rev. Lett. 107, 047003 (2011).

${ }^{68}$ C. Kim, P. J. White, Z.-X. Shen, T. Tohyama, Y. Shibata, S. Maekawa, B. O. Wells, Y. J. Kim, R. J. Birgeneau, and M. A. Kastner, Phys. Rev. Lett. 80, 4245 (1998).

69 J.L. Tallon and J.W. Loram, Physica C 349, 53 (2001).

70 T. Morinari, J. Phys. Soc. Japan 78, 054708 (2009);

71 A. A. Katanin and A. P. Kampf, Phys. Rev. Lett. 93, 106406 (2004)

72 L. Dell'Anna and W. Metzner, Phys. Rev. B 73, 045127 (2006). 
73 M. Abdel-Jawad, M. P. Kennett, L. Balicas, A. Carrington, A. P. Mackenzie, R. H. McKenzie, and N. E. Hussey, Nature Phys. 2, 821 (2006).

74 M. Abdel-Jawad, J. G. Analytis, L. Balicas, A. Carrington, J. P. H. Charmant, M. M. J. French, and N. E. Hussey, Phys. Rev. Lett. 99, 107002 (2007).

75 M.M. J. French, J. G. Analytis, A. Carrington, L. Balicas, and N. E. Hussey, New J. Phys. 11, 055057 (2009).

76 A. Kaminski, S. Rosenkranz, H.M. Fretwell, Z. Z. Li, H. Raffy, M. Randeria, M. R. Norman, and J. C. Campuzano, Phys. Rev. Lett. 90, 207003 (2003).

77 H. Castro and G. Deutscher, Phys. Rev. B 70, 174511 (2004).

78 R. Daou, N. Doiron-Leyraud, D. LeBoeuf, S. Y. Li, F. Laliberté, O. Cyr-Choinière, Y. J. Jo, L. Balicas, J.-Q. Yan, J.-S. Zhou, J. B. Goodenough, and L. Taillefer, Nat. Phys. 5, 31 (2009).

${ }^{79}$ H. Yamase and W. Metzner, Phys. Rev. Lett. 108, 186405 (2012).

80 N. Lin, E. Gull, and A. Millis, Phys. Rev. B 82, 045104 (2010).

81 G. Rickayzen, Green Functions and Condensed Matter, Academic Press (1991).

82 E. Dagotto and J. Riera, Phys. Rev. Lett. 70, 682 (1993).

83 S. S. Kancharla, B. Kyung, D. Sénéchal, M. Civelli, M. Capone, G. Kotliar, and A.-M. S. Tremblay, Phys. Rev. B 77, 184516 (2008).

${ }^{84}$ S. Sorella, G. B. Martins, F. Becca, C. Gazza, L. Capriotti, A. Parola, and E. Dagotto, Phys. Rev. Lett. 88, 117002 (2002).

85 E. Gull, O. Parcollet, and A. Millis, Phys. Rev. Lett. 110, 216405 (2013).

${ }^{86}$ P. A. Lee, T. M. Rice, and P. W. Anderson, Phys. Rev. Lett. 31, 462 (1973);

87 R.A. Cooper, Y. Wang, B. Vignolle, O. J. Lipscombe, S. M. Hayden, Y. Tanabe, T. Adachi, Y. Koike, M. Nohara, H. Takagi, Cyril Proust, and N. E. Hussey, Science 323, 603 (2009).

88 B. P. Xie, K. Yang, D.W. Shen, J. F. Zhao, H.W. Ou, J. Wei, S.Y. Gu, M. Arita, S. Qiao, H. Namatame, M.
Taniguchi, N. Kaneko, H. Eisaki, K. D. Tsuei, C. M. Cheng, I. Vobornik, J. Fujii, G. Rossi, Z. Q. Yang, and D. L. Feng, Phys. Rev. Lett. 98, 147001 (2007).

89 W. Meevasana, X. J. Zhou, S. Sahrakorpi, W. S. Lee, W. L. Yang, K. Tanaka, N. Mannella, T. Yoshida, D. H. Lu, Y. L. Chen, R. H. He, Hsin Lin, S. Komiya, Y. Ando, F. Zhou, W. X. Ti, J. W. Xiong, Z. X. Zhao, T. Sasagawa, T. Kakeshita, K. Fujita, S. Uchida, H. Eisaki, A. Fujimori, Z. Hussain, R. S. Markiewicz, A. Bansil, N. Nagaosa, J. Zaanen, T. P. Devereaux, and Z.-X. Shen, Phys. Rev. B 75, 174506 (2007).

90 J. Graf, G.-H. Gweon, K. McElroy, S.Y. Zhou, C. Jozwiak, E. Rotenberg, A. Bill, T. Sasagawa, H. Eisaki, S. Uchida, H. Takagi, D.-H. Lee, and A. Lanzara, Phys. Rev. Lett. 98, 067004 (2007).

91 W. Zhang, G. Liu, J. Meng, L. Zhao, H. Liu, X. Dong, W. Lu, J. S. Wen, Z. J. Xu, G. D. Gu, T. Sasagawa, G. Wang, Y. Zhu, H. Zhang, Y. Zhou, X. Wang, Z. Zhao, C. Chen, Z. Xu, and X. J. Zhou, Phys. Rev. Lett. 101, 017002 (2008).

92 A. Koitzsch, S. V. Borisenko, A. A. Kordyuk, T. K. Kim, M. Knupfer, J. Fink, H. Berger, and R. Follath, Phys. Rev. B 69, 140507(R) (2004).

93 P.V. Bogdanov, A. Lanzara, S. A. Kellar, X. J. Zhou, E. D. Lu, W. J. Zheng, G. Gu, J.-I. Shimoyama, K. Kishio, H. Ikeda, R. Yoshizaki, Z. Hussain, and Z. X. Shen, Phys. Rev. Lett. 85, 2581 (2000).

94 A. A. Kordyuk, S.V. Borisenko, V. B. Zabolotnyy, J. Geck, M. Knupfer, J. Fink, B. Büchner, C. T. Lin, B. Keimer, H. Berger, A.V. Pan, S. Komiya, and Y. Ando, Phys. Rev. Lett. 97, 017002 (2006).

95 J. Hwang, T. Timusk, and G. D. Gu, J. Phys. Cond. Matter 19, 125208 (2007).

96 Y. Li, M. Le Tacon, M. Bakr, D. Terrade, D. Manske, R. Hackl, L. Ji, M. K. Chan, N. Barišić, X. Zhao, M. Greven, and B. Keimer, Phys. Rev. Lett. 108, 227003 (2012).

97 J. Kokalj and R. H. McKenzie, Phys. Rev. Lett. 107, 147001 (2011).

98 M.M. Zemljič, P. Prelovšek, and T. Tohyama, Phys. Rev. Lett. 100, 036402 (2008). 